\title{
Light Higgs bosons in the general NMSSM
}

\author{
Kiwoon Choi ${ }^{1, a}$, Sang Hui Im ${ }^{2, b}$, Kwang Sik Jeong ${ }^{2, c}$, Chan Beom Park ${ }^{1, \mathrm{~d}}$ \\ ${ }^{1}$ Center for Theoretical Physics of the Universe, Institute for Basic Science (IBS), Daejeon 34051, South Korea \\ ${ }^{2}$ Department of Physics, Pusan National University, Busan 46241, South Korea
}

Received: 1 July 2019 / Accepted: 9 November 2019 / Published online: 21 November 2019

(C) The Author(s) 2019

\begin{abstract}
Physics beyond the Standard Model (SM) may manifest itself as small deviations from the SM predictions for Higgs signal strengths at $125 \mathrm{GeV}$. Then, a plausible and interesting possibility is that the Higgs sector is extended and at the weak scale there appears an additional Higgs boson weakly coupled to the SM sector. Combined with the LEP excess in $e^{+} e^{-} \rightarrow Z(h \rightarrow b \bar{b})$, the diphoton excess around $96 \mathrm{GeV}$ recently reported by CMS may suggest such a possibility. We examine if those LEP and CMS excesses can be explained simultaneously by a singlet-like Higgs boson in the general next-to-minimal supersymmetric Standard Model (NMSSM). Higgs mixing in the NMSSM relies on the singlet coupling to the MSSM Higgs doublets and the higgsino mass parameter, and thus is subject to the constraints on these supersymmetric parameters. We find that the NMSSM can account for both the LEP and CMS excesses at $96 \mathrm{GeV}$ while accommodating the observed $125 \mathrm{GeV}$ SM-like Higgs boson. Interestingly, the required mixing angles constrain the heavy doublet Higgs boson to be heavier than about $500 \mathrm{GeV}$. We also show that the viable region of mixing parameter space is considerably modified if the higgsino mass parameter is around the weak scale, mainly because of the Higgs coupling to photons induced by the charged higgsinos.
\end{abstract}

\section{Contents}

1 Introduction . . . . . . . . . . . . 1

2 Higgs bosons in the general NMSSM . . . . . . 2

2.1 Dependence of Higgs mixing on NMSSM parameters ................ 2

2.2 Effective Higgs couplings to the SM sector . . . 4

3 Mixing consistent with the $125 \mathrm{GeV}$ Higgs boson . . 4

4 LEP and CMS excesses around $96 \mathrm{GeV}$. . . . . . 5

\footnotetext{
ae-mail: kchoi@ibs.re.kr

be-mail: imsanghui@pusan.ac.kr

c e-mail: ksjeong@pusan.ac.kr

de-mail: cbpark@ibs.re.kr
}

5 Summary .................. 8

References . . . . . . . . . . . . . . 9

\section{Introduction}

Although the Standard Model (SM) successfully describes the observed particle physics up to energy scales around $\mathrm{TeV}$, it is clear that a more fundamental theory is needed to provide a complete description of nature. So far the LHC has seen no clear signal for physics beyond the SM, and the discovered $125 \mathrm{GeV}$ Higgs boson has properties compatible with the SM predictions [1,2]. Yet an interesting possibility is that the Higgs sector is extended to include an additional light Higgs boson which is accessible to collider experiments and result in some deviations of the $125 \mathrm{GeV}$ Higgs boson from the SM predictions. The CMS has recently announced that Higgs searches in the diphoton final state show a local excess of about $3 \sigma$ at $96 \mathrm{GeV}$ [3]. The results from the ATLAS do not show a relevant excess, but are well compatible with the CMS limit [4]. Combined with the $2.3 \sigma$ local excess observed in the LEP searches for $e^{+} e^{-} \rightarrow Z(h \rightarrow b \bar{b})[5,6]$, the CMS results provide a motivation to consider the possibility that the Higgs sector involves an additional scalar boson at $96 \mathrm{GeV}$, which has been studied recently in Refs. [7-17].

In this paper we explore if the next-to-minimal supersymmetric SM (NMSSM) can explain the LEP and CMS excesses around $96 \mathrm{GeV}$ while accommodating the observed $125 \mathrm{GeV}$ Higgs boson. The NMSSM extends the Higgs sector to include a gauge singlet scalar which generates the higgsino mass parameter $\mu$ via its coupling $\lambda$ to the MSSM Higgs doublets [18,19]. As noticed in Refs. [20,21], there are intriguing relations between Higgs mixings and the model parameters $\lambda$ and $\mu$ that hold for the general NMSSM. Those relations are quite useful when examining how much Higgs mixings, which determine how the neutral Higgs bosons couple to SM particles [22-29], are constrained by the requirements on the model such as radiative corrections to the Higgs masses, the 
perturbativity bound on $\lambda$, and the chargino mass limit on $\mu$. The viable region of mixing parameter space would be further constrained if one specifies singlet self-interactions. For instance, there are no tadpole and quadratic terms for the singlet in the $Z_{3}$-symmetric NMSSM, ${ }^{1}$ for which the mixing between the neutral singlet and doublet Higgs bosons has a dependence on the mass of the CP-odd singlet scalar.

Our analysis is based on the relations between Higgs mixings and the model parameters, and is performed for the general NMSSM without specifying singlet self-interactions. We first examine if a singlet-like Higgs boson at $96 \mathrm{GeV}$ can be responsible for the LEP and CMS excesses within the range of mixing angles allowed by the current LHC data on the $125 \mathrm{GeV}$ Higgs boson, under the assumption that the gauginos, squarks and sleptons are heavy enough, above $\mathrm{TeV}$ as indicated by the LHC searches for supersymmetry (SUSY), while the higgsinos can be significantly lighter. We then impose the constraints on $\lambda$ and $\mu$ to find the viable mixing angles. It turns out that the general NMSSM can accommodate the SM-like $125 \mathrm{GeV}$ Higgs boson compatible with the current LHC data, and also a singlet-like $96 \mathrm{GeV}$ Higgs boson explaining both the LEP and CMS excesses. The allowed range of mixing angles is considerably modified if $\mu$ is around the weak scale because the charged higgsinos enhance the Higgs coupling to photons. Interestingly, if the excesses around $96 \mathrm{GeV}$ are due to the singlet-like Higgs boson, the heavy doublet Higgs boson should be heavier than about $500 \mathrm{GeV}$.

This paper is organized as follows. In Sect. 2, we briefly discuss the effects of the neutral Higgs boson mixings on Higgs phenomenology and examine the relations between the mixing angles and the NMSSM parameters. The region of the mixing parameter space compatible with the current LHC data on the SM-like Higgs boson is presented in Sect. 3. Section 4 is devoted to our main results, which show the mixing angles required to explain the LEP and CMS excesses, while satisfying the various constraints on the NMSSM parameters. It is also shown that the allowed mixing angles constrain the heavy Higgs boson to have a mass in a certain range. The final section is for the summary and comments.

\section{Higgs bosons in the general NMSSM}

In this section we describe how the neutral Higgs boson mixings depend on the NMSSM parameters, in particular, on the singlet coupling $\lambda$ to the MSSM Higgs doublets and the higgsino mass parameter $\mu$. Such relations should be taken into

\footnotetext{
1 The possibility of accommodating both LEP and CMS excesses in the $Z_{3}$-symmetric NMSSM was firstly explored in Ref. [13]. Our study considers the general NMSSM without any additional symmetry or matter.
}

account when examining the Higgs mixings consistent with the experimental constraints. We also discuss the properties of Higgs bosons within the low energy effective theory constructed by integrating out heavy superparticles under the assumption that the higgsinos can be light. Note that our approach is applicable to a general form of NMSSM.

\subsection{Dependence of Higgs mixing on NMSSM parameters}

Taking an appropriate redefinition of superfields, one can always write the superpotential of the general NMSSM as

$W=\lambda S H_{u} H_{d}+f(S)+($ MSSM Yukawa terms $)$,

with a canonical Kähler potential. Here $H_{u}$ and $H_{d}$ are the Higgs doublet superfields, and $S$ is the gauge singlet superfield. There are various types of NMSSM, depending on the form of the singlet superpotential $f(S)$. Our subsequent discussion applies for a general form of $f(S)$, but for simplicity we will assume no $\mathrm{CP}$ violation in the Higgs sector.

After the electroweak symmetry breaking, the $\mathrm{CP}$-even neutral Higgs bosons

$$
\left(\begin{array}{c}
\hat{h} \\
\hat{H} \\
\hat{s}
\end{array}\right)=\sqrt{2}\left(\begin{array}{ccc}
\sin \beta & \cos \beta & 0 \\
-\cos \beta & \sin \beta & 0 \\
0 & 0 & 1
\end{array}\right)\left(\begin{array}{c}
\operatorname{Re} H_{u}^{0}-v \sin \beta \\
\operatorname{Re} H_{d}^{0}-v \cos \beta \\
\operatorname{Re} S-\langle S\rangle
\end{array}\right)
$$

mix with each other due to the potential terms

$$
\begin{aligned}
V_{\text {mix }}= & \lambda^{2}|S|^{2}\left(\left|H_{u}\right|^{2}+\left|H_{d}\right|^{2}\right)+\left(A_{\lambda} \lambda S H_{u} H_{d}\right. \\
& \left.+\left(\partial_{S} f\right)^{*} \lambda H_{u} H_{d}+\text { h.c. }\right),
\end{aligned}
$$

where $\left\langle H_{u}^{0}\right\rangle=v \sin \beta$ and $\left\langle H_{d}^{0}\right\rangle=v \cos \beta$ with $v=174$ $\mathrm{GeV}$, and $A_{\lambda}$ is the SUSY breaking trilinear coupling. With the above Higgs potential terms, the effective Higgs $\mu$ and $B \mu$ parameters are given by

$$
\begin{aligned}
\mu & =\lambda\langle S\rangle, \\
B \mu & =A_{\lambda} \lambda\langle S\rangle+\lambda\left\langle\partial_{S} f\right\rangle .
\end{aligned}
$$

The supersymmetric parameters $\lambda$ and $\mu$, on which the Higgs mixing depends, are subject to the perturbativity bound and the chargino mass bound, respectively. Imposing the conditions for the electroweak symmetry breaking, the mass 
squared matrix for $(\hat{h}, \hat{H}, \hat{s})$ reads $^{2}$

$\hat{M}_{11}^{2}=m_{0}^{2}+\left(\lambda^{2} v^{2}-m_{Z}^{2}\right) \sin ^{2} 2 \beta$,

$\hat{M}_{12}^{2}=\hat{M}_{21}^{2}=\frac{1}{2}\left(m_{Z}^{2}-\lambda^{2} v^{2}\right) \sin 4 \beta+\Delta m_{12}^{2}$,

$\hat{M}_{13}^{2}=\hat{M}_{31}^{2}=\lambda v(2 \mu-\Lambda \sin 2 \beta)$,

$\hat{M}_{22}^{2}=\frac{2|B \mu|}{\sin 2 \beta}-\left(\lambda^{2} v^{2}-m_{Z}^{2}\right) \sin ^{2} 2 \beta+\Delta m_{22}^{2}$,

$\hat{M}_{23}^{2}=\hat{M}_{32}^{2}=\lambda v \Lambda \cos 2 \beta$,

with $\Lambda$ defined by

$\Lambda \equiv A_{\lambda}+\left\langle\partial_{S}^{2} f\right\rangle$.

Here $\hat{M}_{11}^{2}, \hat{M}_{12}^{2}$, and $\hat{M}_{22}^{2}$ include radiative corrections, which can be sizable as arising from top and stop loops [30]:

$$
\begin{aligned}
m_{0}^{2} \simeq & m_{Z}^{2}+\frac{3 v^{2} y_{t}^{4}}{4 \pi^{2}}\left\{\ln \left(\frac{M_{S}^{2}}{m_{t}^{2}}\right)+\frac{X_{t}^{2}}{M_{S}^{2}}\left(1-\frac{X_{t}^{2}}{12 M_{S}^{2}}\right)\right\}, \\
\Delta m_{12}^{2} \simeq & -\frac{3 v^{2} y_{t}^{4}}{4 \pi^{2} \tan \beta} \\
& \left\{\ln \left(\frac{M_{S}^{2}}{m_{t}^{2}}\right)+\frac{X_{t}\left(X_{t}+Y_{t}\right)}{2 M_{S}^{2}}-\frac{X_{t}^{3} Y_{t}}{12 M_{S}^{4}}\right\}, \\
\Delta m_{22}^{2} \simeq & \frac{3 v^{2} y_{t}^{4}}{4 \pi^{2} \tan ^{2} \beta}\left\{\ln \left(\frac{M_{S}^{2}}{m_{t}^{2}}\right)+\frac{X_{t} Y_{t}}{M_{S}^{2}}\left(1-\frac{X_{t} Y_{t}}{12 M_{S}^{2}}\right)\right\},
\end{aligned}
$$

with $X_{t}=A_{t}-\mu \cot \beta$ and $Y_{t}=A_{t}+\mu \tan \beta$, where $M_{S}=\sqrt{m_{\tilde{t}_{1}} m_{\tilde{t}_{2}}}$ is the geometric mean of the two stop masseigenvalues, and $A_{t}$ is the SUSY breaking trilinear coupling associated with the top quark Yukawa $y_{t}=m_{t} / v$. Note that $m_{0}$ corresponds to the SM-like Higgs boson mass at large $\tan \beta$ in the decoupling limit of the MSSM. The LHC results constrain the stops to be heavier than $\mathrm{TeV}$, and thus $m_{0}$ cannot be lower than about $115 \mathrm{GeV}$ as long as stop mixing has $X_{t}^{2} \lesssim$ $10 M_{S}^{2}$ as is the case in the conventional mediation schemes of SUSY breaking. The stop loop corrections maximizes $m_{0}$ at $X_{t}= \pm \sqrt{6} M_{S}$, i.e. for maximal stop mixing. On the other hand, the charged Higgs boson has a mass around the squareroot of $\hat{M}_{22}^{2}$, and it should be heavier than about $350 \mathrm{GeV}$ to avoid the experimental constraint associated with $b \rightarrow s \gamma$ [31].

2 The value of $\hat{M}_{33}^{2}$ is determined by the singlet superpotential $f(S)$ and the associated soft SUSY breaking terms $F(S)$ according to

$$
\begin{aligned}
\hat{M}_{33}^{2}= & \left(\partial_{S}^{2} f\right)^{2}+\left(\partial_{S} f-\frac{1}{2} \lambda v^{2} \sin 2 \beta\right)\left(\partial_{S}^{3} f-\frac{\partial_{S}^{2} f}{S}\right) \\
& +\frac{1}{2} \lambda v^{2} \frac{A_{\lambda}}{S} \sin 2 \beta+\left(\partial_{S}^{2} F-\frac{\partial_{S} F}{S}\right),
\end{aligned}
$$

where all the terms are evaluated at the vacuum.
To find the mass eigenstates, one needs to diagonalize the mass squared matrix as

$U \hat{M}^{2} U^{\mathrm{T}}=\operatorname{diag}\left(m_{h}^{2}, m_{H}^{2}, m_{s}^{2}\right)$,

where the orthogonal mixing matrix $U$ can be parametrized as

$U=\left(\begin{array}{ccc}c_{\theta_{1}} c_{\theta_{2}} & -s_{\theta_{1}} & -c_{\theta_{1}} s_{\theta_{2}} \\ s_{\theta_{1}} c_{\theta_{2}} c_{\theta_{3}}-s_{\theta_{2}} s_{\theta_{3}} & c_{\theta_{1}} c_{\theta_{3}} & -c_{\theta_{2}} s_{\theta_{3}}-s_{\theta_{1}} s_{\theta_{2}} c_{\theta_{3}} \\ s_{\theta_{1}} c_{\theta_{2}} s_{\theta_{3}}+s_{\theta_{2}} c_{\theta_{3}} & c_{\theta_{1}} s_{\theta_{3}} & c_{\theta_{2}} c_{\theta_{3}}-s_{\theta_{1}} s_{\theta_{2}} s_{\theta_{3}}\end{array}\right)$

with $s_{\theta} \equiv \sin \theta$ and $c_{\theta} \equiv \cos \theta$, for which the angles $\theta_{1}, \theta_{2}$ and $\theta_{3}$ represent $\hat{h}-\hat{H}, \hat{s}-\hat{h}$ and $\hat{s}-\hat{H}$ mixing, respectively. Obviously each matrix element of $\hat{M}^{2}$ can be expressed in terms of the mass eigenvalues $\left\{m_{h}, m_{H}, m_{s}\right\}$ and the mixing angles $\left\{\theta_{1}, \theta_{2}, \theta_{3}\right\}$. Among such relations, the following ones are particularly relevant for our subsequent discussion:

$$
\begin{aligned}
m_{0}^{2}= & m_{h}^{2}+U_{21}\left(U_{21}+U_{22} \tan 2 \beta\right)\left(m_{H}^{2}-m_{h}^{2}\right) \\
& -U_{31}\left(U_{31}+U_{32} \tan 2 \beta\right)\left(m_{h}^{2}-m_{s}^{2}\right) \\
& -\Delta m_{12}^{2} \tan 2 \beta, \\
\lambda v \mu= & \frac{1}{2} U_{23}\left(U_{21}+U_{22} \tan 2 \beta\right)\left(m_{H}^{2}-m_{h}^{2}\right) \\
& -\frac{1}{2} U_{33}\left(U_{31}+U_{32} \tan 2 \beta\right)\left(m_{h}^{2}-m_{s}^{2}\right), \\
\lambda^{2} v^{2}= & m_{Z}^{2}-\frac{2}{\sin 4 \beta}\left(U_{21} U_{22}\left(m_{H}^{2}-m_{h}^{2}\right)\right. \\
& \left.-U_{31} U_{32}\left(m_{h}^{2}-m_{s}^{2}\right)-\Delta m_{12}^{2}\right),
\end{aligned}
$$

because the constraints on the parameters $\lambda, \mu$ and $m_{0}$ can be converted into those on the Higgs boson masses and mixing angles, and also vice versa. Here one should note that $\Delta m_{12}^{2}$ in Eq. (2.11) is written

$\Delta m_{12}^{2}=-\frac{m_{0}^{2}-m_{Z}^{2}}{\tan \beta}+\epsilon v^{2}$,

with $\epsilon$ given by

$\epsilon=-\frac{3 y_{t}^{4}}{8 \pi^{2}} \frac{\mu}{M_{S}}\left(1+\frac{1}{\tan ^{2} \beta}\right)\left(\frac{X_{t}}{M_{S}}-\frac{X_{t}^{3}}{6 M_{S}^{3}}\right)$.

The above shows that $\epsilon$ vanishes at $X_{t}=0$ and $X_{t}=$ $\pm \sqrt{6} M_{S}$, and therefore $\Delta m_{12}^{2}$ is tightly correlated with $m_{0}$ near the regions of minimal and maximal stop mixing. The correlation of the stop corrections in large regions of parameter space has been noted in Ref. [30]. It is also easy to see that $|\epsilon|$ is smaller than about 0.1 for $X_{t}^{2} \lesssim 10 M_{S}^{2}$. For stop mixing with $X_{t}$ far from 0 or $\pm \sqrt{6} M_{S}$, the $\epsilon$-contribution to $\Delta m_{12}^{2}$ can be sizable only when $|\mu|$ is large, close to $M_{S}$, and $\tan \beta$ is low. Keeping this feature in mind, we shall neglect the $\epsilon$-contribution in our analysis unless stated otherwise. 


\subsection{Effective Higgs couplings to the SM sector}

At energy scales around the electroweak scale, the properties of the Higgs bosons can be examined within an effective theory constructed by integrating out heavy sparticles. The LHC results on the Higgs sector and the searches for new physics indicate that SUSY, if exists, would be broken at a scale above TeV. Taking this into account, we assume that all gauginos, squarks and sleptons have masses above TeV, while the higgsinos and additional Higgs bosons can be lighter than $\mathrm{TeV}$. Then the effective lagrangian describing how the neutral Higgs bosons interact with the SM fermions and gauge bosons is written as [32]

$$
\begin{aligned}
\mathcal{L}_{\text {eff }}= & C_{V}^{i}\left(\frac{\sqrt{2} m_{W}^{2}}{v} \phi_{i} W_{\mu}^{+} W^{-\mu}+\frac{m_{Z}^{2}}{\sqrt{2} v} \phi_{i} Z_{\mu} Z^{\mu}\right) \\
& -C_{f}^{i} \frac{m_{f}}{\sqrt{2} v} \phi_{i} \bar{f} f \\
& +\Delta C_{g}^{i} \frac{\alpha_{s}}{12 \sqrt{2} \pi v} \phi_{i} G_{\mu \nu}^{a} G^{a \mu \nu} \\
& +\Delta C_{\gamma}^{i} \frac{\alpha}{\sqrt{2} \pi v} \phi_{i} F_{\mu \nu} F^{\mu \nu},
\end{aligned}
$$

where $\left(\phi_{1}, \phi_{2}, \phi_{3}\right)=(h, H, s)$ and $f$ denotes the SM fermions.

At tree level, the Higgs couplings to massive SM particles are given by

$$
\begin{aligned}
& C_{V}^{i}=U_{i 1}, \quad C_{t}^{i}=U_{i 1}-U_{i 2} \cot \beta, \\
& C_{b}^{i}=C_{\tau}^{i}=U_{1 i}+U_{i 2} \tan \beta .
\end{aligned}
$$

The Higgs couplings to gluons and photons are radiatively generated, which results in

$\Delta C_{g}^{i} \simeq A_{1 / 2}\left(\tau_{t}^{i}\right) C_{t}^{i}+A_{1 / 2}\left(\tau_{b}^{i}\right) C_{b}^{i}+\delta C_{g}^{i}$,

$\Delta C_{\gamma}^{i} \simeq \frac{2}{9} A_{1 / 2}\left(\tau_{t}^{i}\right) C_{t}^{i}-\frac{7}{8} A_{1}\left(\tau_{W}^{i}\right) C_{V}^{i}+\delta C_{\gamma}^{i}$,

where $\delta C_{g}^{i}$ and $\delta C_{\gamma}^{i}$ are additional contributions from sparticle loops, and the loop functions are given by

$$
\begin{aligned}
A_{1 / 2}(\tau) & =\frac{3}{2 \tau^{2}}\{(\tau-1) f(\tau)+\tau\}, \\
A_{1}(\tau) & =\frac{1}{7 \tau^{2}}\left\{3(2 \tau-1) f(\tau)+3 \tau+2 \tau^{2}\right\},
\end{aligned}
$$

where $\tau_{j}^{i} \equiv m_{\phi}^{2} /\left(4 m_{j}^{2}\right)$ and

$f(\tau)= \begin{cases}\arcsin ^{2} \sqrt{\tau} & \text { for } \tau \leq 1 \\ -\frac{1}{4}\left(\ln \frac{1+\sqrt{1-\tau^{-1}}}{1-\sqrt{1-\tau^{-1}}}-i \pi\right)^{2} & \text { for } \tau>1\end{cases}$

For the case when the superpartners of SM particles are heavier than TeV, sparticles give negligible contributions to $\Delta C_{g}^{i}$. However, if $\mu$ is small, the charged higgsinos are light and can give a sizable contribution to $\Delta C_{\gamma}^{i}$ through the following
Higgs-higgsino couplings

$\frac{\lambda}{\sqrt{2}} \sum_{i} U_{i 3} \phi_{i} \tilde{H}_{u}^{+} \tilde{H}_{d}^{-}$

Under the assumption that the gauginos are significantly heavier than the higgsinos, the Higgs coupling to photons induced by charginos can be approximated to be

$\delta C_{\gamma}^{i} \simeq \frac{\lambda v}{6|\mu|}\left(1+\frac{7}{30} \frac{m_{\phi_{i}}^{2}}{4|\mu|^{2}}\right) U_{i 3}$

for a Higgs boson with $m_{\phi_{i}}^{2} \ll 4|\mu|^{2}$.

\section{Mixing consistent with the $125 \mathrm{GeV}$ Higgs boson}

For small scalar mixing, $h$ has properties close to those of the SM Higgs boson. In this paper, we identify $h$ with the SM-like Higgs boson observed at the LHC and examine how the scalar mixing is constrained by the measured signal strengths. The SM-like Higgs boson has $m_{h}=125 \mathrm{GeV}$, and its couplings to the massive SM particles are given by

$$
\begin{aligned}
& C_{V}^{h}=c_{\theta_{1}} c_{\theta_{2}}, \quad C_{t}^{h}=c_{\theta_{1}} c_{\theta_{2}}+s_{\theta_{1}} \cot \beta, \\
& C_{b}^{h}=C_{\tau}^{h}=c_{\theta_{1}} c_{\theta_{2}}-s_{\theta_{1}} \tan \beta,
\end{aligned}
$$

while the couplings to gluons and photons read

$\Delta C_{g}^{h} \simeq 0.97 c_{\theta_{1}} c_{\theta_{2}}+(1.03 \cot \beta+0.06 \tan \beta) s_{\theta_{1}}$,

$\Delta C_{\gamma}^{h} \simeq-0.81 c_{\theta_{1}} c_{\theta_{2}}+0.23 s_{\theta_{1}} \cot \beta-\frac{r}{6} c_{\theta_{1}} s_{\theta_{2}}$.

Here $r$ is defined by

$r \equiv \frac{\lambda v}{|\mu|}$

and measures the chargino contribution, which has been approximated by using the fact that it is non-negligible only when $|\mu|$ is not far above the electroweak scale for $\lambda$ below the perturbative bound, and the chargino search at LEP requires $|\mu|>104 \mathrm{GeV}$. One should note that the couplings of the SM-like Higgs boson are determined by four parameters: $\theta_{1}$, $\theta_{2}, r$ and $\tan \beta$.

The partial decay rates of the SM-like Higgs boson $h$ can be easily estimated by using the well-known decay properties of the hypothetical Higgs boson $\phi_{125}$ of the minimal SM with mass $125 \mathrm{GeV}$ :

$$
\begin{aligned}
\frac{\Gamma(h \rightarrow b b)}{\Gamma\left(\phi_{125} \rightarrow b b\right)} & =\frac{\Gamma(h \rightarrow \tau \tau)}{\Gamma\left(\phi_{125} \rightarrow \tau \tau\right)}=\left|C_{b}^{h}\right|^{2}, \\
\frac{\Gamma(h \rightarrow W W)}{\Gamma\left(\phi_{125} \rightarrow W W\right)} & =\frac{\Gamma(h \rightarrow Z Z)}{\Gamma\left(\phi_{125} \rightarrow Z Z\right)}=\left|C_{V}^{h}\right|^{2}, \\
\frac{\Gamma(h \rightarrow g g)}{\Gamma\left(\phi_{125} \rightarrow g g\right)} & =\frac{\left|\Delta C_{g}^{h}\right|^{2}}{\left|\Delta C_{g}^{\phi_{125}}\right|^{2}},
\end{aligned}
$$




$$
\frac{\Gamma(h \rightarrow \gamma \gamma)}{\Gamma\left(\phi_{125} \rightarrow \gamma \gamma\right)}=\frac{\left|\Delta C_{\gamma}^{h}\right|^{2}}{\left|\Delta C_{\gamma}^{\phi_{125}}\right|^{2}} .
$$

Assuming that $h$ does not decay to non-SM particles, one also finds its total decay rate to be

$$
\frac{\Gamma_{\text {tot }}(h)}{\Gamma_{\text {tot }}\left(\phi_{125}\right)} \simeq 0.64\left|C_{b}^{h}\right|^{2}+0.12\left|C_{t}^{h}\right|^{2}+0.24\left|C_{V}^{h}\right|^{2},
$$

with $\Gamma_{\text {tot }}\left(\phi_{125}\right)$ being the total decay width of $\phi_{125}$. Here we have used the branching ratios of the SM Higgs boson listed in Ref. [33]. The production of the SM-like Higgs boson is dominated by the gluon fusion process, and the signal strength normalized by the SM value is given by

$$
\begin{aligned}
\mu_{h}^{V V} & =\frac{\sigma(p p \rightarrow h)}{\sigma\left(p p \rightarrow \phi_{125}\right)} \frac{\operatorname{Br}(h \rightarrow V V)}{\operatorname{Br}\left(\phi_{125} \rightarrow V V\right)} \\
& \simeq \frac{0.94\left|\Delta C_{g}^{h}\right|^{2}\left|C_{V}^{h}\right|^{2}+0.12\left|C_{V}^{h}\right|^{4}}{0.64\left|C_{b}^{h}\right|^{2}+0.12\left|C_{t}^{h}\right|^{2}+0.24\left|C_{V}^{h}\right|^{2}},
\end{aligned}
$$

for the inclusive $W W / Z Z$ channel, where $\operatorname{Br}(h \rightarrow i i)$ is the branching ratio of the indicated mode. For other channels, one obtains

$$
\begin{aligned}
& \frac{\mu_{h}^{b b}}{\mu_{h}^{V V}}=\frac{\mu_{h}^{\tau \tau}}{\mu_{h}^{V V}}=\frac{\left|C_{b}^{h}\right|^{2}}{\left|C_{V}^{h}\right|^{2}}, \\
& \frac{\mu_{h}^{\gamma \gamma}}{\mu_{h}^{V V}}=\frac{\left|\Delta C_{\gamma}^{h}\right|^{2}}{\left|\Delta C_{\gamma}^{\phi_{125}}\right|^{2}\left|C_{V}^{h}\right|^{2}} \simeq \frac{1.52\left|\Delta C_{\gamma}^{h}\right|^{2}}{\left|C_{V}^{h}\right|^{2}} .
\end{aligned}
$$

It is obvious that one should have $\mu_{h}^{i i}=1$ if $\theta_{1}=\theta_{2}=0$ and $r=0$.

The ATLAS collaboration has recently updated the measurements on the Higgs signal strengths using the $13 \mathrm{TeV}$ data $[1]:^{3}$

$\mu_{h}^{Z Z}=1.13 \pm 0.13$,

and for the other channels

$$
\begin{array}{ll}
\frac{\mu_{h}^{W W}}{\mu_{h}^{Z Z}}=0.84_{-0.15}^{+0.18}, & \frac{\mu_{h}^{\gamma \gamma}}{\mu_{h}^{Z Z}}=0.87_{-0.12}^{+0.14}, \\
\frac{\mu_{h}^{\tau \tau}}{\mu_{h}^{Z Z}}=0.86_{-0.22}^{+0.26}, & \frac{\mu_{h}^{b b}}{\mu_{h}^{Z Z}}=0.84_{-0.27}^{+0.38} .
\end{array}
$$

Note that the NMSSM leads to $\mu_{h}^{W W}=\mu_{h}^{Z Z}$, which is within the $1 \sigma$ range.

Let us now examine how severely the Higgs mixing is constrained by the LHC experimental results on the Higgs boson at $125 \mathrm{GeV}$. The signal rates of $h$ are determined by

\footnotetext{
3 Both ATLAS and CMS collaborations have recently reported their analyses results on the Higgs coupling measurements using the LHC Run 2 data [1,2]. The ATLAS analysis used the larger amount of the Run 2 data, up to the integrated luminosity of $80 \mathrm{fb}^{-1}$. As there is no combined global fit for the full Run 2 data yet, we adopted only the ATLAS result in our study.
}

two mixing angles $\theta_{1}$ and $\theta_{2}$ for given values of $r$ and $\tan \beta$. For instance, $\mu_{h}^{V V}=1$ is obtained if $\theta_{1}$ and $\theta_{2}$ satisfy ${ }^{4}$

$\theta_{1} \approx \frac{\tan \beta}{1.4 \tan ^{2} \beta+1.7} s_{\theta_{2}}^{2}$,

for which the branching ratio for $h \rightarrow V V$ is suppressed compared to the case of the SM Higgs boson, but such effect is compensated by the enhancement of production rate via the gluon fusion process. Note that $r$, which is relevant for the diphoton signal strength, is below about 1.2 because $\lambda$ should be smaller than about 0.7 in order for the NMSSM to remain perturbative up to the GUT scale, and $|\mu|$ should be larger than $104 \mathrm{GeV}$ to satisfy the LEP bound on the chargino mass.

Figure 1 shows the $2 \sigma$ range of $\left(\theta_{1}, \theta_{2}\right)$ allowed by the current LHC data on the Higgs boson. The left panel is for $r=0.1$, for which the Higgs coupling to photons is rarely affected by the charged higgsinos, and the right panel is for $r=1$. Here we have taken $1.5 \leq \tan \beta \leq 15$ to see how the allowed region changes with $\tan \beta$. From the figure, one can see that the allowed region of $\theta_{1}$ gets smaller if $\tan \beta$ increases, but a broad range of $\theta_{2}$ is allowed insensitively to $\tan \beta$. This is because the Higgs coupling $C_{b}^{h}$ gets sensitive to $\theta_{1}$ at large $\tan \beta$ while the couplings to the top quark and gauge bosons do not. If $r$ is around unity, the charged higgsinos can significantly enhance the diphoton signal rate, excluding $\theta_{2}$ in the range between about 0.4 and 0.8 . We can understand this feature from the fact that the charged higgsinos induce a Higgs coupling to photons, $\delta C_{\gamma}^{h} \approx-0.17 r \theta_{2}$ for small mixing angles, whereas the Higgs couplings to other SM particles only quadratically depend on $\theta_{2}$.

\section{LEP and CMS excesses around $96 \mathrm{GeV}$}

The CMS collaboration has recently reported a local excess of $2.8 \sigma$ in the diphoton channel around $m_{\gamma \gamma}=96 \mathrm{GeV}$ [3]. The signal strength amounts to

$\mu_{\mathrm{CMS}}=\frac{\sigma(p p \rightarrow \varphi)}{\sigma\left(p p \rightarrow \phi_{96}\right)} \frac{\operatorname{Br}(\varphi \rightarrow \gamma \gamma)}{\operatorname{Br}\left(\phi_{96} \rightarrow \gamma \gamma\right)} \simeq 0.6 \pm 0.2$,

where $\phi_{96}$ denotes the hypothetical SM Higgs boson with mass $96 \mathrm{GeV}$, and $\operatorname{Br}(\varphi \rightarrow \gamma \gamma)$ denotes the branching ratio for the diphoton channel [13]. Intriguingly, there is another $2.3 \sigma$ local excess at the similar mass region from

4 Although we will not pursue in this paper, a large value of $\theta_{1}$ satisfying

$\theta_{1} \approx \frac{2.1-1.9 s_{\theta_{2}}^{2}}{\tan \beta}$

can also lead to $\mu_{h}^{V V}=1$. In this case, $C_{b}^{h}$ is negative and thus leads to wrong sign Yukawa couplings [20], and one needs a large $\lambda$ beyond the perturbativity bound [34]. 
Fig. 1 Mixing angles $\left(\theta_{1}, \theta_{2}\right)$ compatible with the current LHC data on the $125 \mathrm{GeV}$ Higgs boson for $r=0.1$ (left) and $r=1$ (right), respectively. The shaded region is allowed for $1.5 \leq \tan \beta \leq 15$. The gray and yellow colors show how the allowed region changes with $\tan \beta$

Fig. 2 Mixing angles to explain the LEP and CMS excesses for $r=0.1$ and $r=1$ in the left and right panels, respectively, under the conditions $\lambda<0.7$ and $|\mu|>104 \mathrm{GeV}$. The shaded region is compatible with the observed $125 \mathrm{GeV}$ Higgs boson for $1.5 \leq \tan \beta \leq 15$ as noticed in Fig. 1. The singlet-like Higgs boson with mass $96 \mathrm{GeV}$ can account for the LEP and CMS excesses simultaneously in the red region
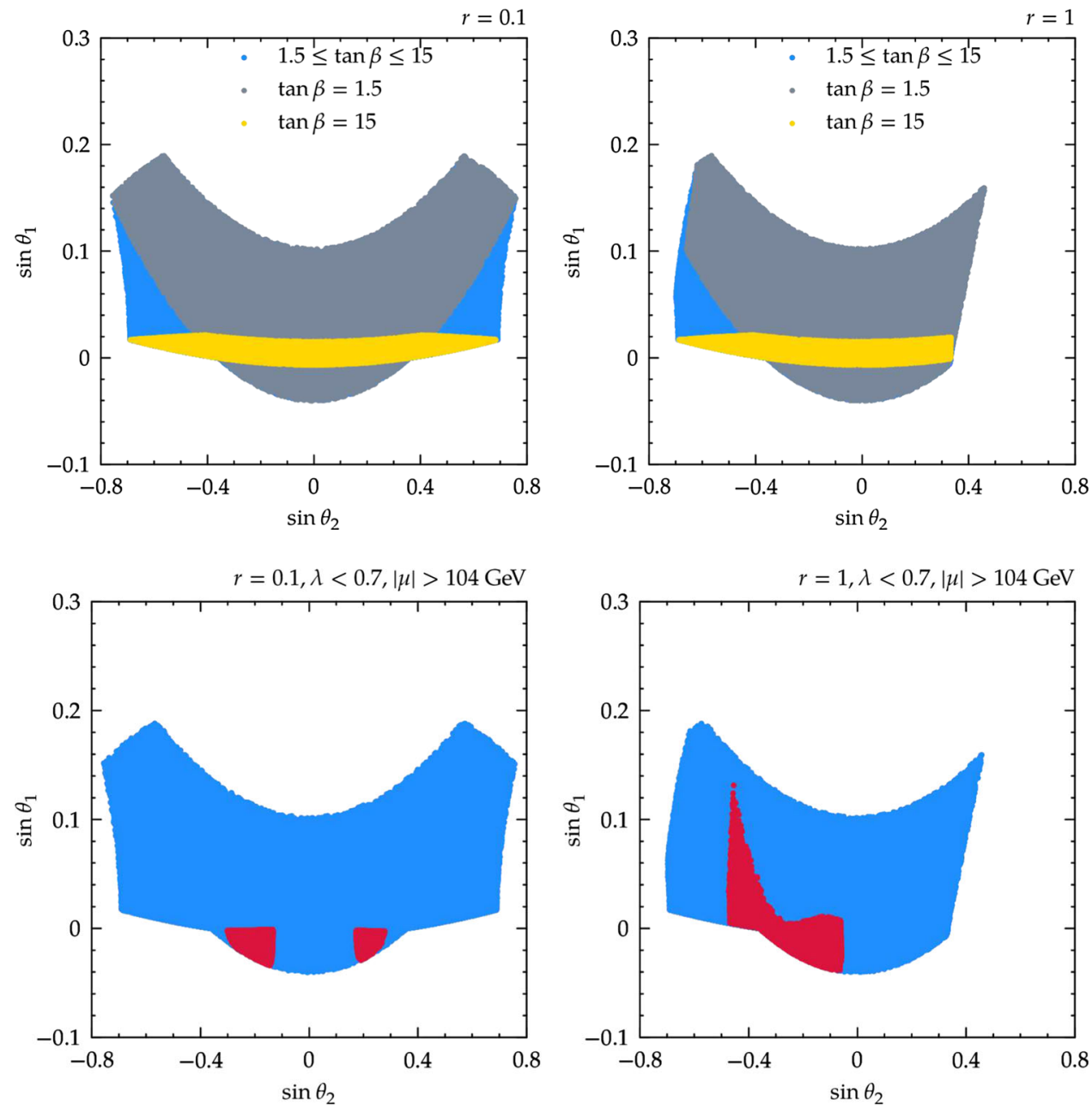

the Higgs searches in the $Z$-boson associated Higgs production $\left(e^{+} e^{-} \rightarrow Z \varphi\right)$ at LEP [5]. The signal strength is [35]

$$
\begin{aligned}
\mu_{\mathrm{LEP}} & =\frac{\sigma\left(e^{+} e^{-} \rightarrow Z \varphi\right)}{\sigma\left(e^{+} e^{-} \rightarrow Z \phi_{96}\right)} \frac{\operatorname{Br}(\varphi \rightarrow b \bar{b})}{\operatorname{Br}\left(\phi_{96} \rightarrow b \bar{b}\right)} \\
& \simeq 0.117 \pm 0.057 .
\end{aligned}
$$

It has long been known that the LEP excess can be explained by a light singlet-like Higgs boson in the NMSSM. At this stage, a naturally occurring question is whether this singletlike Higgs boson can explain the CMS diphoton excess as well.

If both excesses were arisen due to the light singlet-like Higgs boson $s$, the signal strengths can be expressed by the effective couplings in Eq. (2.14) as follows,

$$
\begin{aligned}
\mu_{\mathrm{CMS}} & =\frac{\sigma(p p \rightarrow s)}{\sigma\left(p p \rightarrow \phi_{96}\right)} \frac{\operatorname{Br}(s \rightarrow \gamma \gamma)}{\operatorname{Br}\left(\phi_{96} \rightarrow \gamma \gamma\right)} \\
& \simeq \frac{\left|\Delta C_{g}^{s}\right|^{2}\left|\Delta C_{\gamma}^{s}\right|^{2}}{0.42\left|C_{b}^{s}\right|^{2}+0.05\left|C_{t}^{s}\right|^{2}}, \\
\mu_{\mathrm{LEP}} & =\frac{\sigma\left(e^{+} e^{-} \rightarrow Z s\right)}{\sigma\left(e^{+} e^{-} \rightarrow Z \phi_{96}\right)} \frac{\operatorname{Br}(s \rightarrow b \bar{b})}{\operatorname{Br}\left(\phi_{96} \rightarrow b \bar{b}\right)}
\end{aligned}
$$

$$
\simeq \frac{\left|C_{V}^{s}\right|^{2}\left|C_{b}^{s}\right|^{2}}{0.89\left|C_{b}^{s}\right|^{2}+0.11\left|C_{t}^{s}\right|^{2}},
$$

assuming that the $\mathrm{CP}$-odd singlet scalar and the singlino are heavy enough so that $s$ decays only into the SM particles. Here we have used HDECAY [36,37] to calculate the decay widths of the $96 \mathrm{GeV}$ Higgs boson with the SM couplings. The effective couplings of $s$ are written in terms of the mixing angles as

$$
\begin{aligned}
C_{V}^{s} & =s_{\theta_{1}} c_{\theta_{2}} s_{\theta_{3}}+s_{\theta_{2}} c_{\theta_{3}}, \\
C_{t}^{s} & =s_{\theta_{1}} c_{\theta_{2}} s_{\theta_{3}}+s_{\theta_{2}} c_{\theta_{3}}-c_{\theta_{1}} s_{\theta_{3}} \cot \beta, \\
C_{b}^{s} & =C_{\tau}^{s}=s_{\theta_{1}} c_{\theta_{2}} s_{\theta_{3}}+s_{\theta_{2}} c_{\theta_{3}}+c_{\theta_{1}} s_{\theta_{3}} \tan \beta,
\end{aligned}
$$

and those to gluons and photons read

$$
\begin{aligned}
& \Delta C_{g}^{s} \simeq 1.02 C_{t}^{s}-0.08 C_{b}^{s}, \\
& \Delta C_{\gamma}^{s} \simeq 0.23 C_{t}^{s}-0.96 C_{V}^{s}+\frac{r}{6} U_{33},
\end{aligned}
$$

including the contribution from the loops of charged higgsinos.

Before performing a numerical analysis, we present approximate analytic relations between mixing angles hold- 
Fig. 3 Mixing angles to explain the LEP and CMS excesses for different values of $r$, continued from Fig. 2. Each color shows how the viable region of mixing parameter space is modified when the indicated constraint is imposed

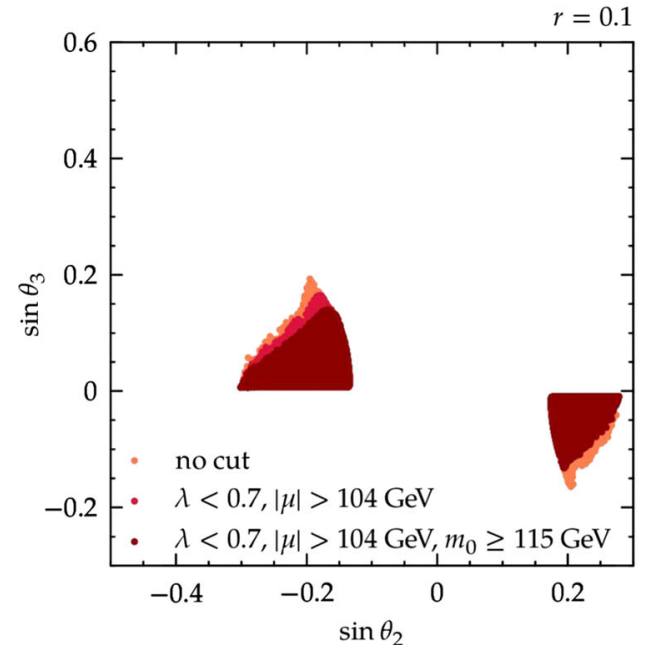

ing if $s$ is responsible for the LEP and CMS excesses. The LEP signal rate given in Eq. (4.4) is approximated by

$\mu_{\mathrm{LEP}} \approx s_{\theta_{2}}^{2}$,

for small $\theta_{2}$, and thus the LEP excess is explained if $s_{\theta_{2}}^{2} \sim 0.1$. On the other hand, the ratio between the CMS and LEP signal rates is given by

$$
\begin{aligned}
\frac{\mu_{\mathrm{CMS}}}{\mu_{\mathrm{LEP}}} \approx & \left(1-k_{32}(1.1 \cot \beta+0.1 \tan \beta)\right)^{2} \\
& \times\left(\frac{1+0.32 k_{32} \cot \beta-0.24 s_{\theta_{2}}^{-1} r}{1+k_{32} \tan \beta}\right)^{2},
\end{aligned}
$$

where $k_{32} \equiv s_{\theta_{3}} / s_{\theta_{2}}$, and it should be around 6 to account for the LEP and CMS excesses. Here the first factor of the right-hand side represents the Higgs production dominated by gluon fusion, while the second one concerns the branching ratio for the diphoton mode. Note that both effects are enhanced if $k_{32}$ is negative, and the charged higgsinos can further increase the branching ratio into photons for $s_{\theta_{2}}<0$. These features help to understand the numerical analysis given below.

Let us explain in detail how to search the viable region of mixing angles in the parameter scan. The signal rates of $h$ and $s$ are functions of the mixing angles, a combination of $\lambda$ and $\mu$, and $\tan \beta$ :

$$
\begin{aligned}
\mu_{h}^{i i} & =\mu_{h}^{i i}\left(\theta_{1}, \theta_{2}, r, \tan \beta\right), \\
\mu_{\mathrm{LEP}, \mathrm{CMS}} & =\mu_{\mathrm{LEP}, \mathrm{CMS}}\left(\theta_{1}, \theta_{2}, \theta_{3}, r, \tan \beta\right),
\end{aligned}
$$

for $r \equiv \lambda v /|\mu|$, with $m_{h}=125 \mathrm{GeV}$ and $m_{s}=96 \mathrm{GeV}$. On the other hand, the relations (2.11) exhibit how the parameters $\lambda, \mu$ and $m_{0}$ change with the mixing angles

$$
\begin{aligned}
\lambda & =\lambda\left(\theta_{1}, \theta_{2}, \theta_{3}, m_{H}, \tan \beta\right), \\
\mu & =\mu\left(\theta_{1}, \theta_{2}, \theta_{3}, m_{H}, \tan \beta\right), \\
m_{0} & =m_{0}\left(\theta_{1}, \theta_{2}, \theta_{3}, m_{H}, \tan \beta\right),
\end{aligned}
$$

where we have taken $\epsilon=0$ in Eq. (2.12) since it is negligibly small in most of the parameter space of our interest. It is obvious that $m_{H}$ is determined by $\theta_{1,2,3}$ and $\tan \beta$ once we fix $r$. The above relations allow us to analyze the viable mixing angles as follows. We first examine the $\left(\theta_{1}, \theta_{2}\right)$ space to see in which region $\mu_{h}^{i i}$ are consistent with the current LHC data, and then continue to check if it is further possible to explain both $\mu_{\mathrm{LEP}}$ and $\mu_{\mathrm{CMS}}$.

In Fig. 2, the Higgs signal rates $\mu_{h}^{i i}$ are consistent with the measurements in the shaded region, and the excesses at $96 \mathrm{GeV}$ are explained in the red shaded region. Figure 3 shows the region of $\theta_{3}$ for the excesses at $96 \mathrm{GeV}$. The next thing that one has to examine is if the viable region above is consistent also with the various constraints on $\lambda, \mu$, and $m_{0}$. Here we have imposed the conditions

$\lambda<0.7, \quad|\mu|>104 \mathrm{GeV}, \quad m_{0} \geq 115 \mathrm{GeV}$,

as required by the perturbativity up to the GUT scale, the LEP limit on the chargino mass, and the radiative contribution to the Higgs mass from stops above $\mathrm{TeV}$, respectively, provided that stop mixing is not too large as would be the case in the conventional mediation models of SUSY breaking. ${ }^{5}$ Then it follows $r \leq 1.1$. Note that $r$ parameterizes the radiative effect of the charged higgsinos on Higgs decays. As benchmark points, we have taken $r=0.1,1$ for $1.5 \leq \tan \beta \leq 15 .{ }^{6}$ Each color in Fig. 3 represents how much the above constraints reduce the viable region. We note that the bound on $m_{0}$ gets important when $\tan \beta$ is small and $r$ is around 1 or above.

In the parameter region for the LEP and CMS excesses, the main effect of stop loop corrections $\Delta m_{12}^{2}$ is to increase (decrease) the coupling $\lambda$ if $\Delta m_{12}^{2}$ is negative (positive),

\footnotetext{
5 The lower bound on $m_{0}$ is set by considering the numerical result that $m_{0}$ is around $113 \mathrm{GeV}$ for stops at $1 \mathrm{TeV}$ in the limit of vanishing stop mixing [38-40].

6 We have cross checked the results of our analysis for some parameter points by using NMSSMTOOls [38-40].
} 
Fig. 4 Region of $\left(\mu, m_{H}\right)$ compatible with the LEP and CMS excesses for $\tan \beta=2$ (left) and 5 (right), respectively. Here we have taken $r$ smaller than 1.1 for $\lambda$ below the perturbativity bound. In each panel, the yellow band is excluded by the LEP results on chargino searches and $m_{0}<115 \mathrm{GeV}$ in the lighter red shaded region. Note that the $m_{0}$ cut is important for $\tan \beta \lesssim 3$
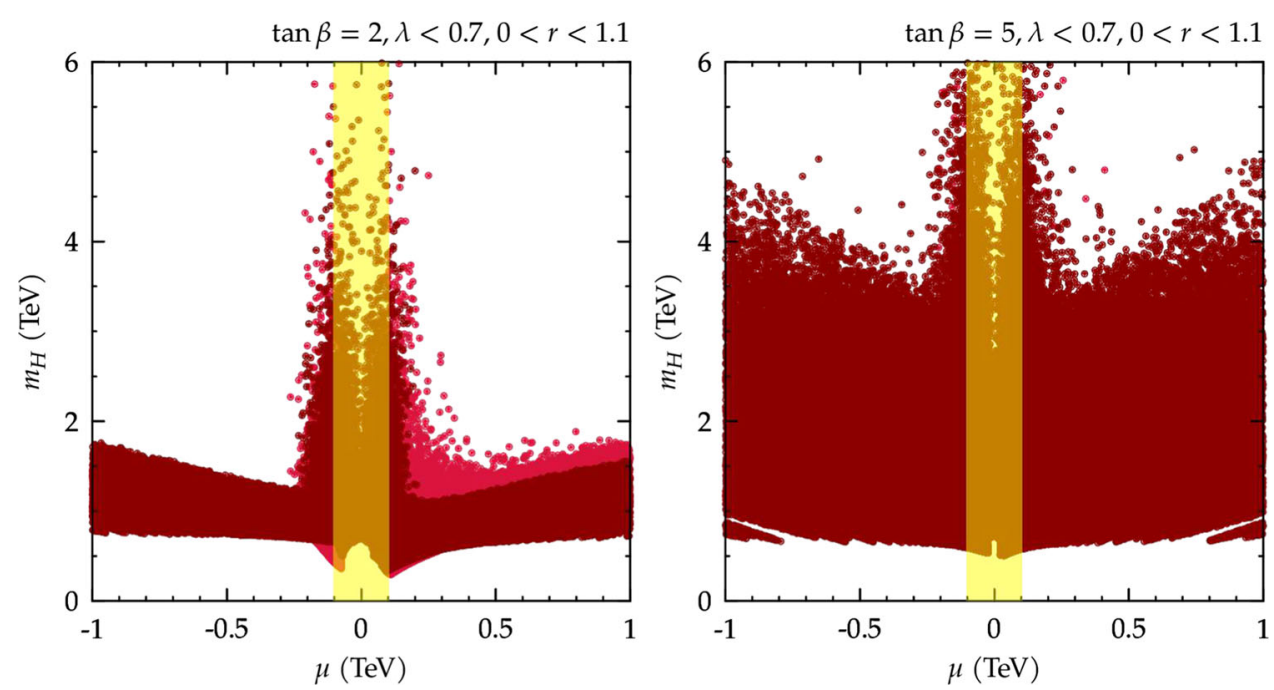

as can be deduced from the last relation in Eq. (2.11). This implies that the parameter space compatible with both excesses shrinks for larger negative $\Delta m_{12}^{2}$ due to the perturbativity bound on $\lambda$. In the parameter region with $m_{0} \geq$ $115 \mathrm{GeV}$, however, the dependence on $\Delta m_{12}^{2}$ becomes quite weak because $\lambda$ should be small in order to get $m_{h}=$ $125 \mathrm{GeV}$. On the other hand, the LEP limit on the chargino mass given in Eq. (4.11) implies $\lambda>0.6 r$, following from $r \equiv \lambda v /|\mu|$. Taking this together with the perturbativity bound on $\lambda$, one can find that $\lambda$ would be more severely constrained at larger $r$ when the stop correction $\Delta m_{12}^{2}$ is negative. We have checked these features by taking analysis for nonzero values of $\epsilon$ between -0.05 and 0.05 .

We close this section by pointing out that the LEP and CMS excesses can constrain the masses of the heavy Higgs boson and higgsinos, if they are due to the singlet-like Higgs boson. Eq. (2.11) enable us to extract the information on the region of $\mu$ and $m_{H}$ compatible with the Higgs signal strengths, $\mu_{h}^{i i}, \mu_{\mathrm{LEP}}$ and $\mu_{\mathrm{CMS}}$. Figure 4 shows the allowed region of $\left(\mu, m_{H}\right)$, where we have taken $\tan \beta=2$ (left) and 5 (right) with $0<r<1.1$. As discussed already, the $m_{0}$ cut is relevant for small $\tan \beta$. It is important to note that the CMS and LEP excesses put a lower and upper bound on $m_{H}$. The lower bound turns out to be $m_{H} \gtrsim 500 \mathrm{GeV}$, nearly irrespectively of the values of $\mu$ and $\tan \beta$, while the upper bound depends on those parameters and is found to increase with $\tan \beta$.

\section{Summary}

Extended with an additional gauge singlet scalar, the Higgs sector of the NMSSM offers a rich phenomenology to be explored at collider experiments. In particular, as experimentally allowed to be light, a singlet-like Higgs boson could be observable in the searches for $e^{+} e^{-} \rightarrow Z(h \rightarrow b \bar{b})$ and $p p \rightarrow h \rightarrow \gamma \gamma$ if it couples to the SM sector via the Higgs mixing. It is thus interesting to examine if the excesses reported by LEP and CMS in those channels can be interpreted as signals of a singlet-like Higgs boson with mass around $96 \mathrm{GeV}$ within the NMSSM.

For the case that the gauginos, squarks and sleptons have masses above $\mathrm{TeV}$, while the Higgsinos can be significantly lighter, which is perfectly consistent with the null results for SUSY searches at LHC so far, we have found that the general NMSSM can successfully accommodate such a light singletlike Higgs boson explaining the LEP and CMS excesses simultaneously, as well as the $125 \mathrm{GeV}$ Higgs boson compatible with the current LHC data. The range of mixing angles required to explain the $96 \mathrm{GeV}$ excesses can be considerably modified if the higgsinos are around the weak scale, because the singlet-like Higgs coupling to photons is enhanced.

To examine a viable region of mixing parameter space, it should be taken into account that Higgs mixing is subject to various constraints on the NMSSM parameters. We have shown that, if a singlet-like Higgs boson is responsible for the LEP and CMS excesses, Higgs mixing is strongly constrained by the LEP bound on the charged higgsino mass and the perturbativity bound on the singlet coupling to the Higgs doublets. Interestingly, in the viable mixing space, the heavy doublet Higgs boson is found to be heavier than about $500 \mathrm{GeV}$.

The physics underlying the electroweak symmetry breaking may manifest itself as slight deviations from the SM predictions for the Higgs signal strengths at $125 \mathrm{GeV}$. It is then a plausible possibility that there exist additional light Higgs bosons weakly coupled to the SM sector, which would provide crucial information on how the Higgs sector is extended. The excesses reported by LEP and CMS, both of which are interestingly around $96 \mathrm{GeV}$, would thus deserve more attention. 
Acknowledgements We would like to thank S. Heinemeyer for useful comments on the manuscript. This work was supported by IBS under the project code, IBS-R018-D1 (KC and CBP), and by the National Research Foundation of Korea (NRF) grant funded by the Korea government (MSIP) (NRF-2018R1C1B6006061) (SHI and KSJ).

Data Availability Statement This manuscript has no associated data or the data will not be deposited. [Authors' comment: There is no data to be deposited because all the analysis results have been obtained by using the analytic equations and experimental inputs included in the article. We believe that interested readers could reproduce the results by codifying the relevant equations.]

Open Access This article is distributed under the terms of the Creative Commons Attribution 4.0 International License (http://creativecomm ons.org/licenses/by/4.0/), which permits unrestricted use, distribution, and reproduction in any medium, provided you give appropriate credit to the original author(s) and the source, provide a link to the Creative Commons license, and indicate if changes were made. Funded by SCOAP ${ }^{3}$.

\section{References}

1. ATLAS Collaboration, Combined measurements of Higgs boson production and decay using up to $80 \mathrm{fb}^{-1}$ of proton-proton collision data at $\sqrt{s}=13 \mathrm{TeV}$ collected with the ATLAS experiment. ATLAS-CONF-2019-005, CERN, Geneva, Mar, 2019. http://cds. cern.ch/record/2668375

2. CMS Collaboration, A.M. Sirunyan et al., Combined measurements of Higgs boson couplings in proton-proton collisions at $\sqrt{s}=13$ TeV.Eur. Phys. J. C 79(5), 421 (2019). arXiv:1809.10733 [hep-ex]

3. CMS Collaboration, A.M. Sirunyan et al., Search for a standard model-like Higgs boson in the mass range between 70 and $110 \mathrm{GeV}$ in the diphoton final state in proton-proton collisions at $\sqrt{s}=8$ and 13 TeV. Phys. Lett. B 793, 320-347 (2019). arXiv:1811.08459 [hep-ex]

4. ATLAS Collaboration, Search for resonances in the 65 to $110 \mathrm{GeV}$ diphoton invariant mass range using $80 \mathrm{fb}^{-1}$ of $p p$ collisions collected at $\sqrt{s}=13 \mathrm{TeV}$ with the ATLAS detector, ATLAS-CONF2018-025, CERN, Geneva, Jul, (2018). http://cds.cern.ch/record/ 2628760

5. LEP Working Group for Higgs boson searches, ALEPH, DELPHI, L3, OPAL Collaboration, R. Barate et al., Search for the standard model Higgs boson at LEP, Phys. Lett. B 565, 61-75 (2003). arXiv:hep-ex/0306033 [hep-ex]

6. ALEPH, DELPHI, L3, OPAL, LEP Working Group for Higgs Boson Searches Collaboration, S. Schael et al., Search for neutral MSSM Higgs bosons at LEP. Eur. Phys. J. C 47, 547-587 (2006). arXiv:hep-ex/0602042 [hep-ex]

7. P.J. Fox, N. Weiner, Light Signals from a Lighter Higgs. JHEP 08 , 025 (2018). arXiv: 1710.07649 [hep-ph]

8. F. Richard, Search for a light radion at HL-LHC and ILC250. arXiv:1712.06410 [hep-ex]

9. U. Haisch, A. Malinauskas, Let there be light from a second light Higgs doublet. JHEP 03, 135 (2018). arXiv:1712.06599 [hep-ph]

10. T. Biekoetter, S. Heinemeyer, C. Munoz, Precise prediction for the Higgs-boson masses in the $\mu \nu$ SSM. Eur. Phys. J. C 78(6), 504 (2018). arXiv:1712.07475 [hep-ph]

11. J.-Q. Tao et al., Search for a lighter Higgs Boson in the Next-toMinimal Supersymmetric Standard Model. Chin. Phys. C 42(10), 103107 (2018). arXiv:1805.11438 [hep-ph]
12. D. Liu, J. Liu, C.E.M. Wagner, X.-P. Wang, A Light Higgs at the LHC and the B-Anomalies. JHEP 06, 150 (2018). arXiv: 1805.01476 [hep-ph]

13. F. Domingo, S. Heinemeyer, S. Passehr, G. Weiglein, Decays of the neutral Higgs bosons into SM fermions and gauge bosons in the $\mathcal{C} \mathcal{P}$-violating NMSSM. Eur. Phys. J. C 78(11), 942 (2018). arXiv:1807.06322 [hep-ph]

14. W.G. Hollik, S. Liebler, G. Moortgat-Pick, S. Passehr, G. Weiglein, Phenomenology of the inflation-inspired NMSSM at the electroweak scale. Eur. Phys. J. C 79(1), 75 (2019). arXiv:1809.07371 [hep-ph]

15. K. Wang, F. Wang, J. Zhu, Q. Jie, The semi-constrained NMSSM in light of muon g-2, LHC, and dark matter constraints. Chin. Phys. C 42(10), 103109-103109 (2018). arXiv:1811.04435 [hep-ph]

16. T. Biekoetter, M. Chakraborti, S. Heinemeyer, A 96 GeV Higgs Boson in the N2HDM. arXiv:1903.11661 [hep-ph]

17. J. M. Cline, T. Toma, Pseudo-Goldstone dark matter confronts cosmic ray and collider anomalies. arXiv:1906.02175 [hep-ph]

18. M. Maniatis, The Next-to-Minimal Supersymmetric extension of the Standard Model reviewed. Int. J. Mod. Phys. A 25, 3505-3602 (2010). arXiv:0906.0777 [hep-ph]

19. U. Ellwanger, C. Hugonie, A.M. Teixeira, The Next-to-Minimal Supersymmetric Standard Model. Phys. Rept. 496, 1-77 (2010). arXiv:0910.1785 [hep-ph]

20. K. Choi, S.H. Im, K.S. Jeong, M. Yamaguchi, Higgs mixing and diphoton rate enhancement in NMSSM models. JHEP 02, 090 (2013). arXiv:1211.0875 [hep-ph]

21. K. Choi, S.H. Im, K.S. Jeong, M.-S. Seo, Higgs phenomenology in the Peccei-Quinn invariant NMSSM. JHEP 01, 072 (2014). arXiv: 1308.4447 [hep-ph]

22. U. Ellwanger, Enhanced di-photon Higgs signal in the Next-toMinimal Supersymmetric Standard Model. Phys. Lett. B 698, 293296 (2011). arXiv:1012.1201 [hep-ph]

23. K.S. Jeong, Y. Shoji, M. Yamaguchi, Singlet-Doublet Higgs Mixing and Its Implications on the Higgs mass in the PQ-NMSSM. JHEP 09, 007 (2012). arXiv:1205.2486 [hep-ph]

24. M. Badziak, M. Olechowski, S. Pokorski, New Regions in the NMSSM with a $125 \mathrm{GeV}$ Higgs. JHEP 06, 043 (2013). arXiv:1304.5437 [hep-ph]

25. K.S. Jeong, Y. Shoji, M. Yamaguchi, Higgs Mixing in the NMSSM and Light Higgsinos. JHEP 11, 148 (2014). arXiv:1407.0955 [hep$\mathrm{ph}]$

26. U. Ellwanger, M. Rodriguez-Vazquez, Discovery Prospects of a Light Scalar in the NMSSM. JHEP 02, 096 (2016). arXiv:1512.04281 [hep-ph]

27. C. Beskidt, W. de Boer, D.I. Kazakov, Can we discover a light singlet-like NMSSM Higgs boson at the LHC? Phys. Lett. B 782, 69-76 (2018). arXiv:1712.02531 [hep-ph]

28. K.S. Jeong, Singlet-like Higgs boson in the NMSSM. J. Korean Phys. Soc. 70(1), 28-33 (2017)

29. L. Liu, H. Qiao, K. Wang, J. Zhu, A Light Scalar in the Minimal Dilaton Model in Light of LHC Constraints. Chin. Phys. C 43(2), 023104 (2019). arXiv:1812.00107 [hep-ph]

30. M. Carena, H.E. Haber, I. Low, N.R. Shah, C.E.M. Wagner, Alignment limit of the NMSSM Higgs sector. Phys. Rev. D 93(3), 035013 (2016). arXiv:1510.09137 [hep-ph]

31. P. Gambino, M. Misiak, Quark mass effects in anti-B to X(s gamma). Nucl. Phys. B 611, 338-366 (2001). arXiv:hep-ph/0104034 [hep-ph]

32. D. Carmi, A. Falkowski, E. Kuflik, T. Volansky, J. Zupan, Higgs after the discovery: a status report. JHEP 10, 196 (2012). arXiv:1207.1718 [hep-ph]

33. LHC Higgs Cross Section Working Group Collaboration, J. R. Andersen et al., Handbook of LHC Higgs Cross Sections: 3. Higgs Properties. arXiv:1307.1347 [hep-ph] 
34. N.M. Coyle, B. Li, C.E.M. Wagner, Wrong sign bottom Yukawa coupling in low energy supersymmetry. Phys. Rev. D 97(11), 115028 (2018). arXiv:1802.09122 [hep-ph]

35. J. Cao, X. Guo, Y. He, P. Wu, Y. Zhang, Diphoton signal of the light Higgs boson in natural NMSSM. Phys. Rev. D 95(11), 116001 (2017). arXiv:1612.08522 [hep-ph]

36. A. Djouadi, J. Kalinowski, M. Spira, HDECAY: A Program for Higgs boson decays in the standard model and its supersymmetric extension. Comput. Phys. Commun. 108, 56-74 (1998). arXiv:hep-ph/9704448 [hep-ph]

37. A. Djouadi, J. Kalinowski, M. Muehlleitner, M. Spira, HDECAY: Twenty $_{++}$years after. Comput. Phys. Commun. 238, 214-231 (2019). arXiv:1801.09506 [hep-ph]
38. U. Ellwanger, J.F. Gunion, C. Hugonie, NMHDECAY: A Fortran code for the Higgs masses, couplings and decay widths in the NMSSM. JHEP 02, 066 (2005). arXiv:hep-ph/0406215 [hep-ph]

39. U. Ellwanger, C. Hugonie, NMHDECAY 2.0: An Updated program for sparticle masses, Higgs masses, couplings and decay widths in the NMSSM. Comput. Phys. Commun. 175, 290-303 (2006). arXiv:hep-ph/0508022 [hep-ph]

40. U. Ellwanger, C. Hugonie, NMSPEC: A Fortran code for the sparticle and Higgs masses in the NMSSM with GUT scale boundary conditions. Comput. Phys. Commun. 177, 399-407 (2007). arXiv:hep-ph/0612134 [hep-ph] 